\title{
Les tribulations de Joseph Rosny (1771-1814) questions sur le statut de l'écrivain en révolution
}

The tribulations of Joseph Rosny (1771-1814). Questions about the status of the writer in Revolution

Jean-Luc Chappey

\section{(2) OpenEdition}

\section{Journals}

Édition électronique

URL : https://journals.openedition.org/ahrf/10625

DOI : 10.4000/ahrf.10625

ISSN : 1952-403X

Éditeur :

Armand Colin, Société des études robespierristes

Édition imprimée

Date de publication : 1 avril 2009

Pagination : 119-142

ISBN : 978-2-200-92558-1

ISSN : 0003-4436

\section{Référence électronique}

Jean-Luc Chappey, «Les tribulations de Joseph Rosny (1771-1814) questions sur le statut de

l'écrivain en révolution », Annales historiques de la Révolution française [En ligne], 356 | avril-juin 2009 mis en ligne le 01 juin 2012, consulté le 23 avril 2022. URL : http://journals.openedition.org/ahrf/ 10625 ; DOI : https://doi.org/10.4000/ahrf.10625 


\title{
LES TRIBULATIONS DE JOSEPH ROSNY (1771-1814) QUESTIONS SUR LE STATUT DE L'ÉCRIVAIN EN RÉVOLUTION
}

Jean-Luc CHAPPEY

\begin{abstract}
En étudiant la trajectoire complexe de Joseph Rosny, il s'agit de s'interroger sur la position complexe de l'homme de lettres en Révolution et sur les différentes actions d'écriture dont ce que l'on a coutume de classer sous la notion de littérature n'est qu'un aspect particulier. Parce que Rosny ne peut être réduit au seul statut "d'écrivain ", il permet de mieux comprendre la place des activités d'écriture dans des stratégies qui dépassent largement les contours du seul " espace littéraire ». II ne s'agit pas de rajouter le nom d'un écrivain inconnu au Panthéon littéraire, mais au final de mieux comprendre les processus à partir desquels des écrivains particulièrement productifs pendant la période révolutionnaire ont pu être progressivement marginalisés et " oubliés » par une histoire littéraire qui, en forgeant des archétypes, ont exclu de fait les acteurs aux identités polymorphes.
\end{abstract}

Mots-clés : écrivain, histoire de la littérature, militaire, identité.

Comme l'a montré Haïm Burstin ${ }^{1}$ pour les sciences, l'étude d'une trajectoire individuelle est sans doute - sans pour autant renoncer aux études qualitatives sur les réseaux et les institutions ${ }^{2}-$ un moyen privilégié

(1) Voir son étude sur Cassini et l'Observatoire dans " "Bohème intellectuelle" et sansculottes », L'invention du sans-culotte, Paris, Odile Jacob, 2005, p. 141-191.

(2) Jean-Claude Bonnet (dir.), La Carmagnole des Muses. L'homme de lettres et l'artiste dans la Révolution, Paris, Armand Colin, 1988 ; idem, L'empire des muses. Napoléon, les arts et les lettres, Paris, Belin, 2004 ; Gregory Brown, Literary Sociability and Literary Property in France, 1775-1793. Beaumarchais, the Société des Auteurs dramatiques and the Comédie française, Londres, 
pour en finir avec l'approche désincarnée des hommes de Lettres et de leurs productions qui alimente encore trop souvent une vision idéalisée - voire héroïque - de l'histoire littéraire ${ }^{3}$. C'est le cas, à bien des égards, des travaux de Nathalie Heinich qui analyse le processus de construction de l'identité des écrivains et de ses transformations à travers les catégories et les représentations construites par les acteurs eux-mêmes ${ }^{4}$; loin de faire jouer les relations que l'écriture entretient avec d'autres formes de statut ou de profession, elle s'interroge sur les modalités fictionnelles ou symboliques de la construction d'un statut. Plus généralement, l'écrivain est toujours étudié à travers des catégories pré-construites qui participent aux illusions de la cohérence telles qu'elles peuvent encore apparaître à travers les usages de la notion de «carrière » littéraire. Faute d'une véritable histoire sociale, l'analyse des écrivains et de leurs productions sous la Révolution française reste ainsi dominée par un double système de représentation. D'une part, on se retrouve face à la figure de «l'homme de Lettres » conçue, dans la continuité des Lumières, comme la conscience critique d'une société et d'une opinion publique dont il représenterait l'avant-garde. Nous retrouvons là l'idée du «sacerdoce laïc » proposée en son temps par Paul Bénichou ${ }^{5}$ dont la théorie sur le «sacre de l'écrivain » reste une référence essentielle, reprise par exemple dans les travaux de Didier Masseau'. Dans une perspective linéaire, l'individualisation et la dénonciation de la dépendance de l'écrivain aux «mécènes » constitueraient des étapes du basculement entre les Lumières et le Romantisme qui verrait s'imposer une nouvelle " grande figure », celle du " génie » incarnée par Chateaubriand. Ce dernier est particulièrement prisé par les biographes qui, à l'instar d'un Marc Fumaroli ${ }^{7}$, ne font souvent que réifier

(Suite de la note 2 page 123)

Ashgate, 2006 ; Antoine LiLTI, Sociabilité et mondanité à Paris au XVIII siècle, Paris, Fayard, 2005 ; Philippe Bourdin et Jean-Luc CHAPPEy (dir.), Réseaux et sociabilités littéraires en Révolution, Presses de l’Université Blaise Pascal, Clermont-Ferrand, 2007.

(3) Notons que depuis quelques années, la biographie participe au renouveau des études sur le monde des Lettres et des écrivains : Michel Vovelle, Théodore Desorgues ou la désorganisation, Paris, Seuil, 1985 ; Jean-Paul Bertaud, Choderlos de Laclos, l'auteur des Liaisons dangereuses, Paris, Fayard, 2003 ; Dominique Margairaz, François de Neufchâteau. Biographie intellectuelle, Paris, Publications de la Sorbonne, 2005.

(4) Nathalie Heinich, Être écrivain. Création et identité, La Découverte, 2000 ; idem, L'élite artiste. Excellence et singularité en régime démocratique, Paris, Gallimard, 2005.

(5) Paul BÉNICHOU, Le sacre de l'écrivain, 1750-1830. Essai sur l'avènement d'un pouvoir spirituel lä̈que dans la France moderne, Paris, José Corti, 1973.

(6) Didier Masseau, L'invention de l'intellectuel dans l'Europe du XVIII siècle, Paris, PUF, 1994.

(7) Marc Fumaroli, Chateaubriand. Poésie et Terreur, Paris, Éditions de Fallois, 2003. 
les catégories et les distinctions construites par les écrivains eux-mêmes pour légitimer leur position et justifier de nouvelles formes de distinction au sein de l'espace littéraire. D'autre part, dans une perspective différente, les écrivains apparaissent encore comme des littérateurs opportunistes et girouettes, prompts à saisir l'événement pour se construire une réputation et vendre leur plume pour célébrer les régimes en place. Considérée comme une période de revanche des "exilés du Parnasse » et autres « Rousseau des ruisseaux $»^{8}$, la période révolutionnaire est par là victime d'une mauvaise réputation, justifiant le désintérêt pour ces «petits" écrivains. Si ces deux systèmes d'interprétation ne sauraient être ignorés, il nous semble nécessaire d'en affiner les présupposés. L'histoire sociale doit permettre de préciser les études des dynamiques qui traversent le monde des Lettres pendant la Révolution française. Les écrivains sont alors rarement exclusivement écrivains, en partie pour des raisons économiques, mais pas uniquement ${ }^{9}$. Contraints de trouver d'autres sources de revenus ou étant investis dans d'autres activités, ils ne peuvent être réduits au statut d'agents stables d'un champ ${ }^{10}$. Considérant que les «stratégies d'auteur $\rangle^{11}$ peuvent largement déborder des contours de l'espace littéraire, il convient de s'interroger sur les diverses actions d'écriture mises en œuvre par un individu, certaines de ces actions pouvant relever de la littérature ${ }^{12}$. Le monde des Lettres ne fonctionne pas en vase clos et les interactions entre les différents terrains où se déplacent les acteurs (administration, édition, armée...) rendent ainsi compte des enjeux qui entourent les stratégies de publication, les logiques de légitimation et des modes de consécration. Ceci implique d'analyser toutes les actions et les représentations de l'acteur sans distinguer a priori celles que l'on aurait spontanément rapportées à l'activité d'homme de Lettres ${ }^{13}$. C'est dans cette perspective que l'étude biographique d'un auteur " oublié et dédaigné » par l'historiographie peut être justifiée. Entre 1794 et 1812, Joseph Rosny

(8) Robert DARNTON, Bohème littéraire et Révolution. Le monde des livres au XVIII siècle, Paris, Gallimard-Le Seuil, 1983.

(9) Cf. sur ce point Bernard LAHIRE, La condition littéraire. Le double vie des écrivains, Paris, La Découverte, «Textes à l'appui/ Laboratoire des sciences sociales », 2006 ; voir la critique de cet ouvrage par Gisèle SAPIRO dans Annales HSS, 62 ${ }^{\mathrm{e}}$ année, ${ }^{\circ}$ 4, juillet-août 2007, p. 947-949.

(10) Pierre Bourdieu, « Le champ littéraire », Actes de la recherche en sciences sociales, sept. 1991, p. 4-46.

(11) Alain Viala, Naissance de l'écrivain. Sociologie de la littérature à l'âge classique, Paris, Éd. de Minuit, 1985.

(12) Nicolas SchapIRA, Un professionnel des lettres au XVII siècle. Valentin Conrart : une histoire sociale, Paris, Champ Vallon, 2003.

(13) Bruno Latour, Changer de société. Refaire de la sociologie, Paris, La Découverte, 2006. 
(1771-1814) publie près de quarante ouvrages caractérisés par leur extrême hétérogénéité (théâtre, romans, récits moralisateurs, dictionnaires, ouvrages érudits, journaux savants...) participant à cette prolifération bibliographique qui caractérise la production des Restif de la Bretonne, Mercier ou madame de Genlis. Est-il possible de trouver une cohérence au parcours bio-bibliographique d'un acteur dont l'identité ne cesse d'échapper aux formes de classifications traditionnelles?

\section{Les conditions d'entrée dans le monde des Lettres (1794 - 1797)}

Jeune noble né à Paris le 29 août 1771, Joseph Rosny s'oriente vers la carrière militaire : à sa sortie du collège militaire de Rebais en 1787, il devient soldat au régiment de Vexin en 1788 puis sergent fourrier (chargé des approvisionnements) en 1789. Cette même année, il publie, suivant les traces d'un Choderlos de Laclos, Ulamor et Feltidie, un roman « féérique » et " allégorique », qui aurait, selon l'auteur, " une action plus puissante que tous les procédés du cher docteur Mesmer $»^{14}$. À la suite de ce coup d'essai dont il est impossible de mesurer la portée, Rosny poursuit sa carrière dans les armes : volontaire en 1791 au $1^{\text {er }}$ bataillon du département du Cher, il devient sous-lieutenant dans les compagnies franches en 1792 ; lieutenant puis quartier maître trésorier de la Légion des Ardennes l'année suivante, il occupe en 1794 les fonctions d'adjoint du général de brigade Jacob et participe aux combats en Vendée. Pour autant, cette carrière militaire ne lui fait pas totalement oublier les Lettres : profitant sans doute de la vague porteuse de l'an II liée aux commandes des autorités en faveur des ouvrages pédagogiques, il publie une Vie de Jean-Pierre Florian qui prend place dans le tome XV des œuvres de «l'ami des enfants ». La période de la Terreur voit l'émergence d'un nombre important d'auteurs nouveaux et occasionnels qui investissent les genres mis à l'honneur à l'époque comme la chanson ou le théâtre ${ }^{15}$. Parmi ceux qui cherchent à profiter des commandes publiques et à se forger un statut d'écrivain « officiel», les militaires occupent une place de choix. Adjoint aux adjudants généraux de l'armée de l'Ouest, Rosny est réformé pour des problèmes de santé en 1795 . Véritable tournant dans sa trajectoire, il entre alors dans l'administration militaire puis civile: sous-chef de la commission des armées de terre en l'an IV/1796, puis commis d'ordre

(14) Joseph Rosny, Ulamor et Feltidie. Histoire allégorique, traduite par M. ${ }^{* *} R$., A Londres et Paris, chez Gattey, libraire de S.A.S. Madame la duchesse d'Orléans, au Palais Royal, 1789, 2 t.

(15) Michel Vovelle, Théodore Desorgues, op. cit., p. 124-125. 
général auprès du ministre de la Police générale Cochon, il devient, en l'an V/1797, sous-chef du Bureau de l'esprit public au même ministère. C'est sans doute à cette époque qu'il épouse la fille de Gabriel-AntoineJoseph Hécart (1755-1838), libraire à Paris, installé au n ${ }^{\circ} 10$ passage des Jacobins Saint-Jacques. Entre administration et librairie, Rosny cherche alors à renforcer sa position dans le monde des Lettres : à partir de 1797, il multiplie les productions investissant en véritable polygraphe les genres les plus différents (biographie, roman, drame...). Or, les activités de Rosny ne sauraient être distinguées, bien au contraire.

Si la production littéraire du Directoire reste encore largement méconnue, cette période se caractérise par un essor important des publications pédagogiques qui, sous des formes et des genres différents, participent à la vaste entreprise de « républicanisation des esprits ». Sous-chef du Bureau de l'esprit public, Rosny profite indéniablement du contexte politique lié à la contre-offensive républicaine pour participer ainsi à cette entreprise, confirmant ainsi les études menées sur François de Neufchâteau ${ }^{16}$ ou sur les différents employés du ministère de l'Intérieur ${ }^{17}$ qui révèlent la forte perméabilité existant entre le monde de l'administration et celui des Lettres pendant le Directoire. Moins visibles que d'autres formes d'interventions des autorités publiques sur le terrain des productions littéraires (commandes, aides à la publication, secours et pensions accordées aux auteurs...), le rôle joué par ces acteurs situés entre le monde des Lettres et le monde de l'administration est pourtant essentiel pour rendre compte des dynamiques qui traversent respectivement ces deux univers. Comme d'autres qui prennent la plume pour défendre et diffuser les principes de la Révolution et de la République par simple opportunisme ou réelle sincérité, Rosny cherche autant à construire son statut d'écrivain qu'à renforcer sa position au sein de la hiérarchie administrative. Après une réédition de la Vie de Florian, il prend place dans les productions « morales » et pédagogiques, investissant les différents genres littéraires (théâtre, mémoires, roman) pour diffuser les principes républicains $^{18}$. Dans des mémoires fictifs publiés en 1797 , les Infortunes de $M^{r}$ de

(16) Dominique Margairaz, François de Neufchâteau, op. cit.

(17) Catherine KawA, Les ronds-de-cuir en Révolution. Les Employés du ministère de l'Intérieur sous la Première République (1792-1800), Paris, CTHS, 1996.

(18) Pensons encore aux auteurs de manuels élémentaires et autres catéchismes politiques étudiés par Jean-Charles ButTIER : Les manuels élémentaires de morale et d'instruction civique pendant la période révolutionnaire (1784-1807). Approche politique, économique et culturelle, mémoire de maîtrise, IHRF/Université de Paris I, Catherine Duprat (dir.), 1997. 
La Galetierre ${ }^{19}$ présentés comme un «monument de honte de barbarie », il multiplie les attaques virulentes contre le personnel de l'an II et réduit la politique menée par le gouvernement révolutionnaire à la violence et la barbarie : « En retraçant au public une partie des horreurs commises sous le règne de la terreur, ce serait tout à la fois l'intéresser et le mettre en garde contre un nouveau système de sang et d'anarchie $»^{20}$. Il publie encore un drame en trois actes - Le régime décemvira ${ }^{21}$ - dans lequel il réitère ses attaques contre le régime terroriste et le personnel administratif de l'an II. Rosny, sans ignorer la mode, justifie sa pratique d'écrivain comme relevant d'une mission au service de la République. Il mobilise ses différents statuts et professions, non seulement pour y puiser les matériaux nécessaires à l'écriture, mais encore pour justifier sa pratique d'écrivain. L'écriture ne sert pas seulement à construire une cohérence à sa trajectoire biographique, mais participe encore à la transformation de sa condition. Ainsi, toujours en 1797, il publie un roman, Joseph et Caroline ou le Berger de la Sologne. Mémoires d'un jeune homme, fidèlement rédigés par lui-même Histoire véritable, par un officier de la légion des Ardennes ${ }^{22}$. Construit à travers la correspondance fictive entre un jeune militaire et un de ses amis, ce roman épistolaire intègre pour une très large part des éléments autobiographiques : au récit de la jeunesse d'un jeune noble en rupture de ban avec son ordre, en conflit avec sa mère au sujet d'un amour impossible, succède la narration des expériences militaires du « héros » qui, engagé volontaire en 1792 dans la Légion des Ardennes, est envoyé ensuite en Vendée pour combattre les rebelles. La deuxième partie du roman pourrait même être considérée comme les mémoires militaires de Rosny ; "L'histoire [ayant] toujours au dessus du roman le mérite de la réalité », il intègre dans son récit des documents administratifs et autres témoignages qui doivent en renforcer la " vérité » et rappeler les sacrifices du narrateur et, plus généralement, des soldats de la République. Alors qu'à la fin du récit, le narrateur évoque le bonheur simple dans lequel il vit, ce calme contraste quelque peu avec la fièvre d'écriture dont semble pourtant être atteint Rosny à partir de 1798 .

(19) Joseph Rosny, Les infortunes de $M^{\mathrm{r}}$ de La Galetierre pendant le régime décemviral contenant ses persécutions, sa fuite sous Robespierre, son naufrage, son séjour... par A.-J. Rosny, Paris, impr. de Conort, rue de la Harpe, 1797, 162 p.

(20) Ibid., p. I.

(21) Joseph Rosny, Le Régime décemviral, fait historique, drame en 3 actes et en prose, par Joseph $R^{* * *}$ [Rosny], Paris, chez tous les marchands de nouveautés, 1797-an V, 126 p.

(22) Joseph Rosny, Joseph et Caroline ou le Berger de la Sologne. Mémoires d'un jeune homme, fidèlement rédigés par lui-même. Histoire véritable, par un officier de la légion des Ardennes, Paris, impr. de Conort, an V-1797. 
Dédicacé à sa femme, son Gernance ou la force des passions. Anecdote français ${ }^{23}$ est encore une histoire édifiante dédiée à la « bonne épouse » et à la famille en montrant la nécessité du mariage et des liens fondés sur la confiance entre époux. Or, à ce moment, Rosny ne figure plus parmi le personnel des bureaux du ministère de l'Intérieur qu'il a quittés au début de l'année. Espère-t-il pouvoir tirer suffisamment de revenus de ces seules productions littéraires ou a-t-il été victime d'une réduction ou d'un changement - fréquent à l'époque - du personnel du ministère ? Là encore, les sources sont silencieuses. Il n'en reste pas moins que Rosny doit désormais faire flèche de tout bois pour faire vivre son foyer grâce à la littérature. En septembre 1798, il publie, en collaboration avec un certain Louis-François Béraud de la Rochelle, un mélodrame en quatre actes «avec danses, chansons, décors et costumes créoles ", Adonis ou le bon nègre qui sera joué sans succès sur la scène de l'Ambigu-comique. C'est désormais à travers la narration des difficultés et autres périls qui se dressent face à ceux qui aspirent au statut d'écrivain que Rosny, jouant encore sur la frontière ambiguë entre fiction et témoignage individuel, cherche à se gagner un public de lecteurs.

\section{Les difficultés d'être écrivain (1798 - 1802)}

Dans son Firmin, ou le jouet de la fortune, histoire d'un jeune $e^{e ́ m i g r e ́}{ }^{24}$, Rosny narre les péripéties d'un jeune homme au moment de la Révolution. Après son arrivée à Paris, « ville immense où chacun ne pense qu'à soi », le jeune héros doit abandonner, face à la concurrence liée au grand nombre d'artistes, l'activité de peintre qu'il exerçait à Liège et choisit de s'orienter vers celle d'écrivain, un choix présenté comme insatisfaisant et déterminé par la nécessité et la contrainte : "Cet état, jadis considéré, cet état, autrefois aussi honorable qu'indépendant, était tombé dans un état d'avilissement qui se ressentait du bouleversement général. Cependant, je n'avais pas de choix à faire ; la carrière littéraire était la seule qui me convînt, la seule qui fût à ma portée $»^{25}$. Le récit livre alors de nombreuses réflexions sur le statut d'écrivain et les différentes contraintes qui pèsent sur lui. Érigeant Florian et l'écriture « champêtre » en modèles, il mesure l'écart entre son idéal d'écriture (incarné dans un

(23) Joseph Rosny, Gernance ou la force des passions. Anecdote française, Paris, chez Le Prieur, Libraire, rue de Savoie, an VI, $142 \mathrm{p}$.

(24) Joseph Rosny, Firmin, ou le Jouet de la fortune, histoire d'un jeune émigré, par Joseph Rosny, Paris, Pigoreau, an VI, 2 parties en 1 vol.

(25) Ibid., p. 131. 
système de notions : « nature », « imagination », « romance », « idylle », « félicité champêtre », « poésie »...) et la médiocrité de ses propres productions. Il oppose ainsi au modèle de l'écrivain désintéressé la condition d'écrivain besogneux qui doit écrire pour vivre, contraire selon lui à la mission «morale » qu'il doit suivre : « Dans ma situation, je n'y pouvais prétendre. En effet, je plains l'homme de Lettres qui est obligé de travailler pour vivre, et d'enchaîner les muses pour soutenir son existence; non seulement ses productions se ressentent de la stérilité de sa bourse, mais encore il est forcé de lutter contre une troupe de vampires affamés qui calculent leur fortune sur sa détresse $»^{26}$. Ces «vampires affamés » ne sont autres que les libraires qui sont les cibles de très violentes critiques. À Paris, seuls quelques-uns («tout au plus une vingtaine ») sont dignes « de la considération dont jouissait l'ancienne librairie » :

«Les autres ne sont à proprement parler, que de misérables brocanteurs qui savent à peine distinguer l'édition originale de la contrefaçon ; cependant la plupart d'entre eux, pour ajouter à leur commerce une branche de plus, se sont rendus éditeurs et même propriétaires des productions de nos meilleurs auteurs : j'en ai connu qui achetaient les manuscrits avec autant d'impudeur qu'ils auraient fait d'un objet qu'ils se seraient procurés au marché ou dans une salle de vente $»^{27}$.

Alors qu'il devient lui-même la cible de critiques portées contre les écrivains qui « inonderaient» le marché de «mauvais » petits livres ${ }^{28}$, il n'en accuse pas moins les libraires et les libertés du commerce d'être responsables de la mauvaise qualité et du déclin des productions littéraires car «les productions d'un auteur infortuné doivent nécessairement se ressentir de sa détresse et de la situation de son esprit $»^{29}$. Firmin/Rosny doit pourtant accepter les conditions de ces « mercenaires » et choisir un genre d'écriture lucratif et garantissant le succès commercial : il s'oriente alors vers le genre romanesque, s'excusant auprès des lecteurs de choisir un genre léger (« le Français, fatigué de politique, ne se piquait pas alors

(26) Ibid., p. 134.

(27) Ibid., p. 135.

(28) On trouvera ainsi une attaque contre Rosny dans Les ombres ou les vivants qui sont morts. Fantasmagorie littéraire. Almanach pour l'an X, Paris, de l'imprimerie de la rue Cassette, an X/1801 : «Rosni - Il fait un roman par décade. Le total de ses ouvrages est par conséquent de trente-six par an. Si cet auteur, encore jeune, vit quarante ans, la littérature sera enrichie de 1440 volumes. Heureusement qu'on est pas obligé de le lire, étant du nom... de ces auteurs manœuvres Dont nul n'est riche assez pour acheter ses œuvres », p. 133.

(29) Joseph Rosny, Firmin, op. cit., p. 146. 
d'érudition ») et dangereux pour les mœurs. Mais face aux difficultés économiques et à la concurrence commerciale, Firmin, à l'image du narrateur, change de stratégie et s'oriente vers l'écriture théâtrale "pour parvenir à la fortune plus promptement et avec plus de certitude $»^{30}$. Il se heurte alors à l'indifférence méprisante des administrateurs de théâtre ${ }^{31}$ et, là encore, doit affronter l'échec :

« Je sentis qu'il était encore plus facile de faire imprimer un mauvais roman, que de faire jouer une bonne comédie. Je fis marché à tant de feuille avec le plus célèbre marchand de nouveautés... J'étais dans la détresse, mon existence dépendait de la quantité de mes productions, et je me décidai à devenir prolixe. Mon génie créateur enfantait par mois des volumes qui tombaient aussitôt dans l'oubli $»^{32}$.

Ses efforts ne suffisent pas à satisfaire ses besoins, bien au contraire. Progressivement, la position de Firmin connaît un véritable déclassement et le narrateur présente la littérature «commerciale» comme une porte ouverte vers la dégradation sociale et morale : «Je sentis d'ailleurs que j'allais me dégrader entièrement, et me perdre pour jamais de réputation. Je rougis de l'état d'avilissement dans lequel je réduisais les lettres, et je m'aperçus qu'il était temps de me frayer un nouveau chemin à la fortune $\|^{33}$. L'entrée dans le monde des Lettres est ainsi présentée comme une « chute». Victime, Firmin est contraint de se servir de l'intrigue, du mensonge, de la tromperie pour parvenir: secrétaire d'un «nouveau riche », il devient l'amant de sa femme ; " ma vertu échoue contre la morale d'une courtisane ; je deviens ingrat, criminel... » confie-t-il. Plus loin, il fait la connaissance d'un journaliste qui, partageant son sort, lui assure que seules les autorités publiques peuvent reconnaître la qualité d'un écrivain et assurer le progrès des Lettres : "Vous n'êtes point heureux; je n'en suis pas surpris, c'est en général le sort des gens de Lettres; ils végètent presque tous, à moins que leurs talents éminents, ou leur supériorité bien reconnue, ne fixent l'attention du gouvernement $»^{34}$. À la descente aux enfers succède la rédemption et l'histoire se finit bien : en quittant le monde des Lettres et en abandonnant l'état d'écrivain,

(30) Ibid., p. 149.

(31) Cf. sur les transformations du monde du théâtre sous le Directoire, Philippe BouRdin, Gérard Loubinoux (dir.), Les arts de la scène et la Révolution française, Clermont-Ferrand, Presses universitaires Blaise-Pascal, 2004.

(32) Joseph Rosny, Firmin, op. cit., p. 169.

(33) Ibid., p. 170.

(34) Ibid., t. II, p. 46. 
Firmin se bâtit une petite fortune honnêtement et se réconcilie avec son père. Si l'on aurait tort de réduire ce roman à une autobiographie, il révèle les formes de représentations et les topiques de l'écrivain auxquels Rosny emprunte certains traits. À la fin du second tome, il introduit un « Avertissement nécessaire ou préface inutile » où il critique le « goût » des lecteurs et les agissements des libraires :

« Je vous ai trompé, mon cher lecteur, j'ai abusé de votre bonne foi en donnant à mon ouvrage un titre fallacieux [...]. C'est un peu votre faute ; pourquoi diable aussi vous avisez-vous de ne vouloir, dans vos lectures, que de grands événements. Convenez à votre tour que si, tout simplement, j'eusse intitulé mon livre, Histoire véritable, que vous ne l'eussiez pas lu, pas même acheté ; et cependant les aventures de Firmin ont le mérite de la réalité. J'ai pu, selon l'usage, embellir mon récit, le broder parfois, mais j'ose affirmer que le fond n'en est pas moins vrai. Cependant, afin que vous n'ayez aucun reproche à me faire, je vous invite, cher lecteur, à le regarder comme un roman absolument dénué de vraisemblance, et pur effet de l'imagination. Il serait même impolitique de ma part de l'annoncer autrement, car ce serait me faire inutilement des ennemis, et me mettre à dos certains libraires qui ne me pardonneraient jamais d'avoir mis au jour le tableau fidèle de leurs petits moyens de spéculations, ainsi que de la juste estime qu'ils méritent $»^{35}$.

Tout en étant particulièrement critique contre la «petite » littérature, Rosny n'en continue pas moins d'explorer les genres de production les plus divers. En 1798, il publie L'optique du jour ou le Foyer de Montan$\operatorname{sier}^{36}$, récit sous forme d'une galerie de portraits servant de support à la description d'une société du Directoire représentée de manière particulièrement négative comme le théâtre de l'intrigue, de la corruption et des fortunes faciles. À travers le parcours d'un jeune provincial qui découvre la société du Directoire, le récit est prétexte à des descriptions souvent satiriques des différents types sociaux, préfigurant ainsi un genre d'enquête sociale particulièrement prisé pendant toute la première moitié du XIX ${ }^{\mathrm{e}}$ siècle ${ }^{37}$. Le lecteur visite ainsi de hauts lieux de la vie politique et culturelle de Paris. On y croise l'agioteur, le rentier, la courtisane, le nouveau parvenu, le joueur, l'anarchiste, le militaire, l'émigré... et bien sûr l'homme de

(35) Ibid., t. II, p. 182-184.

(36) L'optique du jour ou le Foyer de Montansier, par Joseph $R^{* * *} y$, Paris, chez Marchand, Libraire, an VII.

(37) Judith Lyon-CAen, « Saisir, décrire, déchiffrer : les mises en texte du social sous la monarchie de Juillet », Revue historique, CCCVI/2, 2002, p. 303-331. 
Lettres. Là encore, l'évocation du statut des écrivains est particulièrement pessimiste : "Pour les trois quarts d'entre eux, le talent est encore non seulement un moyen de plaire, mais un moyen de vivre...». Le récit s'appesantit sur la description des contrastes sociaux au sein du monde des Lettres, le narrateur portant ses critiques autant sur les " petits» que sur les écrivains illustres. Ainsi, cet auteur « dont l'impudence surpasse encore le talent, est un littérateur distingué, mais qui ressemble à une femme... L'amour propre l'aveugle au point de se croire un géant. Il s'imagine régir de son cabinet le Parnasse en entier et avoir seul le droit de dicter des lois dans les états d'Apollon. Il regarde les débutants dans la carrière littéraire comme autant de Mirmidons ou de Pygmées qui font des efforts impuissants pour se traîner jusqu'au pied du mont sacré... $»^{38}$. Ces attaques, qui s'inscrivent dans une longue tradition, participent alors à la radicalisation des luttes qui traverse, dans un contexte de forte concurrence et de crise du monde de l'édition, le monde des Lettres. Évoquant ces querelles littéraires, l'auteur en montre la vacuité et critique le désordre qui semble exister au sein du monde des Lettres du Directoire : "Ce sont des querelles d'auteurs, et ces sortes de spadassins ne se battent qu'à la plume. Ils en sont quittes pour riposter une flèche à celle qu'on leur lance et plusieurs d'entr'eux s'en font un jeu. Ils appellent ces sortes d'escarmouches les bases de leur réputation $»^{39}$. Néanmoins, en dépit des critiques, le narrateur reconnaît qu'il existe, dans le monde des écrivains, des auteurs plus méritants dont il semble solliciter la protection : «Il en est qui au-dessus de la critique et de l'envie naissant, encouragent le jeune homme qui débute, lui tendent la main protectrice, le soutiennent, le protègent et guident ses pas chancelants et incertains dans la carrière des lettres ». Alors que dans Firmin, il dénonce les effets des déréglementations sociales, institutionnelles et surtout commerciales sur la littérature, il en appelle désormais aux autorités, et plus précisément au ministre de l'Intérieur, pour remettre de l'ordre dans le monde des Lettres. Bonaparte saura bientôt s'appuyer sur ces revendications venues d'acteurs comme Rosny pour légitimer ses interventions de plus en plus fréquentes, conduisant à la remise en place d'une véritable relation de mécénat ${ }^{40}$.

Les appels successifs lancés par Rosny aux autorités ne restent pas sans réponse : protégé par François de Neufchâteau dont il a judicieusement célébré les qualités de ministre et d'homme de Lettres, Rosny - dont

(39) Ibid., p. 126.

(40) Annie Jourdan, Napoléon. Héros, imperator, mécène, Paris, Aubier,1998. 
les compétences littéraires ont assurément été reconnues - devient premier rédacteur à l'Instruction publique ; il rejoint la cohorte des auteurs « subventionnés » réunis autour du ministère de l'Intérieur et issus, selon Dominique Margairaz, du « demi-monde des lettres parisiennes $»^{41}\left(\right.$ Parny ${ }^{42}$, Joseph Lavallée, François Félix Nogaret, La Chabeaussière, Nicolas Bouilly ou Dieudonné Thiébault). Or, après quelques mois d'activité, il quitte l'administration civile pour rejoindre l'administration militaire. Il est élevé au grade de capitaine quartier maître trésorier, chargé par le ministère de la Guerre d'organiser les bataillons de l'Aveyron. Mais le vote de la loi Jourdan entraîne une nouvelle réorganisation de l'armée qui a pour conséquence la réforme de nombreux régiments. Rosny se retrouve de nouveau sans emploi. Il réinvestit alors la littérature, renforçant encore ses attaques contre la dégradation de la littérature et le désordre du monde de la librairie et de l'imprimerie.

Au moment où le Directoire connaît de plus en plus de difficultés, Rosny participe avec bien d'autres à la dénonciation du régime républicain. Alors que dans les journaux se multiplient les attaques contre la politique de soutien accordée par le régime aux hommes de Lettres, contre le rôle assigné à ces derniers dans l'administration ${ }^{43}$ et, finalement, contre la fonction assignée à la littérature dans l'œuvre de régénération républicaine, Rosny s'inscrit activement dans cette offensive contre la « décadence » des Lettres et la « dégradation » de la littérature. En collaboration avec deux littérateurs dont la position est proche de la sienne - Félix Nogaret (1740-1831) et Claude-François-Félix Mercier de Compiègne (1763-1800) -, il publie, quelque temps avant le coup d'État du 18 brumaire, un violent pamphlet contre les « nouveaux venus » et autres « usurpateurs » dans le monde des Lettres. Sous le titre de Tribunal d'Apollon, les auteurs de cet ouvrage se présentent comme les «juges» impartiaux chargés de rétablir les hiérarchies et l'ordre dans la République des Lettres. Dans la continuité du Petit dictionnaire des grands hommes de Rivarol, l'ouvrage se présente comme un dictionnaire satirique où les différentes notices

(41) Dominique Margairaz, François de Neufchâteau, op. cit., p. 364.

(42) Catriona Seth, « Parny et l'instruction publique ; un affrontement d'idéologies », dans P. Bourdin, B. Gainot (dir.), La République directoriale, Paris, Société des études robespierristes, 1998 , t. 2, p. 439-454.

(43) Voir par exemple Louis-Germain Petitain (dit Polichinelle), « Quelques vues sur ce qu'on appelle la propagation des Lumières et les effets naturels d'une grande multiplication de cette classe d'hommes dits philosophes, penseurs, gens de lettres ", Mémoires d'économie publique, Paris, t. 1, n' V, p. 279-313. 
biographiques servent d'exécutoires aux critiques souvent acerbes contre les écrivains en place :

« Ce jourd'hui, les Chambres du Parnasse assemblées à l'effet de juger en dernier ressort, et indistinctement tous les habitants du monde littéraire, ainsi que d'assigner une place aux intrus, aux nouveaux venus, et généralement à tous ceux qui, sans titres, sans moyens, sans y être appelés par une vocation particulière, s'obstineraient à usurper la réputation, les droits et privilèges appartenant aux naturels du pays. Considérant que l'état de dépérissement dans lequel se trouvent les lettres, ne provient que de l'invasion faite depuis quelques temps par des nations voisines et barbares, ou par de ridicules mirmidons et pygmées. Considérant que l'on ne doit attribuer en partie cette décadence qu'à l'admission des diables, spectres et revenants, qui, depuis nombre d'années, obscurcissent l'horizon littéraire. Considérant que la plupart des sujets d'Apollon, aveuglés par la flatterie, l'amour-propre, ou par quelques demi-succès, refusent de se rendre justice, et qu'il devient important de leur assigner sur le Parnasse à chacun la place qui lui est due. Considérant que ni la faveur ni l'intrigue ne doivent avoir aucune part dans cette distribution, et que ces places ne doivent être décernées qu'aux talents et au vrai mérite. Considérant enfin qu'il est temps de régénérer le goût, les mœurs, la saine morale et la philosophie, en ne reconnaissant dans la République des Lettres que ceux qui y ont des droits par leurs travaux, leurs succès, ou leurs dispositions. Le Tribunal se déclarant compétent, malgré l'absence de quelques-uns de ses membres, juge et condamne en dernier ressort, ainsi qu'il suit, les sujets d'Apollon à se ranger aux pieds du Mont-sacré, pour prendre possession du poste qui leur est assigné par le talent $»^{44}$.

Ces dictionnaires, qui servent autant à publier les réputations au sein du monde des Lettres qu'à les détruire, deviennent, entre 1799 et 1802, les principales armes de la guerre qui caractérise le monde des Lettres et dont les auteurs ne cessent justement de dénoncer des « effets » qu'ils ne font qu'exaspérer davantage. Se cristallisant rapidement entre adversaires et partisans des Lumières, cette « guerre» ne peut cependant pas être réduite à un affrontement théorique. Les attaques sont d'autant plus violentes qu'elles prennent pour cible des protagonistes qui peuvent apparaître comme les concurrents directs des auteurs, dans un contexte où

(44) Joseph Rosny et alii, Le Tribunal d'Apollon ou Jugement en dernier ressort de tous les auteurs vivants. Libelle injurieux, partial et diffamatoire par une société de pygmées littéraires, Paris, chez Marchand, an VIII, vol. 1, p. IX-XIII. 
le marasme du marché éditorial conjugué à une baisse des commandes publiques $^{45}$ est particulièrement défavorable pour ceux là mêmes qui avaient cru pouvoir se tailler une réputation d'écrivain en s'appuyant sur le soutien des autorités. Rosny, véritable « forçat» de l'écriture comme le souligne la notice qui lui est consacrée, est particulièrement victime de cette mauvaise conjoncture : « Ses mille et un romans n'ont pas eu mille et un lecteurs; et comme il l'avoue lui-même, il écrit à la toise, à la mesure pour vendre ses œuvres comme on vend des boisseaux de pommes de terres $»^{46}$. Or, une nouvelle occasion s'offre à lui : en décembre 1799, Lucien Bonaparte l'appelle aux fonctions de commis divisionnaire au ministère de l'Intérieur. Alors que le salaire de 6000 livres doit améliorer ses conditions de vie, il est victime de la mise à l'écart de son protecteur en mars 1800 . Rosny est de nouveau sans-emploi. S'affublant des habits du philosophe retiré du monde ${ }^{47}$, il tente encore de répondre aux goûts du jour en publiant un ouvrage sur les plaisirs de la vie à la campagne et des « plaisirs rustiques ».

Dans Le Bonheur rural ${ }^{48}$, il critique la " grande ville » et célèbre la « Nature », " les mœurs et la vie champêtre »; affirmant ne point " écrire pour les partisans de la nouveauté », il dénonce le «penchant» pour les « intrigues invraisemblables » et les « situations gigantesques » et entend - épousant l'air du temps - ramener les lecteurs aux charmes de la « douce philosophie et de la saine morale ». Réaffirmant la mission morale d'une littérature dégagée des contraintes du commerce, il dénonce les « ambitieux » qui « passent leurs jours à courir après la volage fortune et qui, serviles esclaves de leurs passions, soupirent sans cesse après les honneurs et les dignités ». Rosny présente cet ouvrage comme l'ultime tentative pour se faire reconnaître comme écrivain ; après avoir reconnu la « ridicule prétention d'obtenir une place parmi les littérateurs plus âgé » que lui, il déclare : « Si ce dernier essai ne parvient point à me concilier l'estime de mon lecteur; si l'on ne dit de moi, en terminant mon livre : un jour, il pourra faire mieux ; je brise à jamais, j'en fais le serment, je brise le pipeau champêtre, et je promets d'avance d'ensevelir dans un éternel oubli jusqu'au souvenir de mes impuissants efforts $»^{49}$. On sait qu'il

(45) Carla Hesse, Publishing and Cultural Politics in Revolutionary Paris, 1789-1810, University of California Press, Berkeley, 1991, p. 204.

(46) Ibid., p. 216.

(47) Joseph Rosny, Le rêve d'un philosophe ou voici toute mon ambition, Paris, imprimerie de Glisau, an VIII.

(48) Le Bonheur rural, ou Tableau de la vie champêtre, poème en 12 livres et en prose, par Joseph Rosny, Paris, Poisson, 1801, xx-276 p., pl.

(49) Ibid., p. XVIII. 
oubliera très vite ce serment! Quelques mois plus tard, il publie un long roman épistolaire, Le Péruvien à Paris ${ }^{50}$ qui s'inscrit alors dans la vogue de la littérature panoramique ${ }^{51}$. À travers les lettres échangées entre un jeune Péruvien ramené en France par un « vieux philosophe » et sa famille restée au pays, Rosny trouve de nouveau prétexte à peindre quelques tableaux particulièrement précis, et souvent drôles, de la vie dans la capitale et dans les provinces. Le récit s'achève par l'évocation du "génie bienfaisant sous la forme d'un héros » qui vient sauver la France. Si ces preuves d'allégeance ne sont pas sans effet, Rosny sait sans doute surtout conserver des relations susceptibles de jouer en sa faveur dans les moments de réelles difficultés. Recommandé par Lebrun auprès du ministre de l'Intérieur Chaptal, ce dernier le nomme en l'an X (1801) directeur de l'octroi municipal à Autun pour un salaire de 1700 livres. Cette situation n'est pas sans avantages, mais elle l'oblige à quitter Paris. Loin de plaire à celui qui chantait quelques mois plus tôt les " plaisirs champêtres ", il semble que cet éloignement soit vécu rapidement comme un exil. Rosny doit de nouveau se tourner vers les autorités pour tenter de quitter la Bourgogne.

$\mathrm{Au}$ début de l'année 1802, il publie Au gouvernement français ${ }^{52}$ dans lequel il sollicite directement le premier Consul pour obtenir un emploi. Ce texte, qui s'inscrit dans le vaste corpus des lettres relevant des « secours et pensions », est original à de nombreux égards. Par sa publication, d'une part, il rompt avec le circuit «normal» de diffusion de ces lettres ; par sa composition et son contenu, il se situe à la frontière des mémoires individuels (il s'agit d'un long récit de vie) et du document administratif sur le modèle d'un état des services enrichi de pièces justificatives se rapportant à sa carrière militaire. Se présentant comme " homme de Lettres, capitaine réformé et directeur de l'octroi à Autun », Rosny cherche, à travers ce récit qui fait jouer ses qualités littéraires, à rendre cohérente sa trajectoire biographique. C'est sans doute à cette occasion que l'on mesure la complexité de cette "identité de papier» construite par Rosny qui ne saurait être réduite à l'une de ses «professions » : il est finalement très peu question du statut d'homme de Lettres

(50) Joseph Rosny, Le Péruvien à Paris, ouvrage critique, historique et moral contenant la Relation de voyage d'un jeune Indien, fait en France au commencement du dix-neuvième siècle, son entrée dans le monde, ses aventures et ses critiques sur les mœurs, usages, coutumes et établissements des Français, Paris, imprimerie de Huguin, an IX (1801).

(51) Pensons par exemple au succès obtenu par un proche de Rosny, Mercier de Compiègne avec son Manuel du voyageur à Paris (1798).

(52) Joseph Rosny, Au gouvernement français, Autun, imp. de P. P. Dejussieu, an X, 31 p. 
car c'est après avoir largement mis en valeur son statut de militaire, puis d'administrateur, qu'il insiste sur son statut d'écrivain : «Enfin, mes travaux littéraires, en établissant la mesure de mes moyens, suffisent également pour donner une idée précise de mes principes politiques $\rangle^{53}$. À travers cette lettre de sollicitation (appelée peut-être à servir de modèle ?), il cherche en effet en se situant sur tous les terrains, littéraire, militaire et administratif, un moyen de consolider sa position dans un de ces domaines en s'appuyant sur les ressources respectives des deux autres. Il utilise en large partie ses qualités d'écrivain pour construire un récit qu'il espère suffisamment convaincant pour permettre son rappel à Paris dans l'administration civile ou militaire. Revêtant les habits de l'ermite (« Je résolus de rompre avec la société, et je me retirai avec les miens, dans une petite habitation rustique que je me plus à cultiver de mes propres mains $» / \ll$ Loin des hommes, de la fortune et de ses trompeuses faveurs, je passai mes premiers moments entre le culte des Muses et l'éducation de mes enfants $\rangle^{54}$ ), il justifie sa lettre par les problèmes financiers qui semblent l'empêcher de remplir sa mission de «bon » mari et père de famille : «Une épouse et des enfants chéris, une mère âgée et infirme, voilà l'objet de mes tendres sollicitudes; Ne seraient-elles comptées pour rien dans un siècle de lumières, de philanthropie et de vertus sociales ? $\rangle^{55}$. Il justifie le fait de publier ce texte par son isolement et son manque de protection: au-delà d'un besoin de se distinguer dans la « foule des solliciteurs qui, de toutes part, assiègent le Palais du gouvernement par leurs clameurs importunes », Rosny dénonce en effet le système de secours qui est fondé sur les « intrigues », le « secret » : « La justice que je réclame est publique [...]. Je suis a soixante-dix lieues de la Capitale; je n'ai conservé près de vous ni protections, ni intrigues ; l'estime seule peut me servir en vous remettant personnellement ce mémoire : aussi je ne me recommande qu'à l'estime pour plaider auprès de vous la cause de l'infortune $»^{56}$. Multipliant les poncifs, il dénonce l'artifice, le jeu de la mondanité, des flagorneries, des intrigues et des réseaux : « Je n'ai pas à craindre que la multiplicité de vos fonctions importantes ne bannisse de votre souvenir l'homme obscur qui n'est point entouré de ce faux brillant nécessaire à quiconque se hasarde à se produire sans l'appui de quelques protections puissantes $»^{57}$. Dès le

(53) Ibid., p. 18.

(54) Ibid., p. 14.

(55) Ibid., p. 9.

(56) Ibid., p. 20.

(57) Ibid., p. 17. 
début de cette demande, Rosny se place sur le terrain de l'économie du « service » qui nous ramène à son identité nobiliaire et, plus sûrement, à son statut de militaire. C'est au nom des services rendus et des sacrifices réalisés par le militaire, l'administrateur et l'homme de Lettres que Rosny plaide sa cause et attend de cette «juste réclamation » une juste rétribution : " Ce n'est point une grâce, une faveur qu'il se hasarde à réclamer ; ce n'est point une retraite, une pension ni un secours qu'il vient demander à grands cris, mais ce sont les moyens de servir utilement sa patrie... $\rangle^{58}$. Il présente sa situation comme scandaleuse du fait du contraste entre les services rendus et sa condition présente :

« Après avoir rempli avec distinction pendant dix années consécutives, des emplois en chef, tant dans la partie administrative, que dans la partie militaire ; après avoir constamment consacré mes veilles, mes travaux et mes moyens littéraires à la propagation des principes et de la morale, je suis aujourd'hui pour ainsi dire exilé dans une petite ville de provinces, avec le titre de Directeur de l'octroi [or] ce n'est point avec 1700 liv. qu'il est possible d'alimenter une famille nombreuse [...] Ce n'est pas ainsi que vous récompensez les hommes qui ont sacrifié leur fortune, leur jeunesse et leur sang pour concilier le gouvernement républicain ».

L'argument est des plus simples : Rosny n'occupe pas la place qu'il mérite et, par là, la Nation toute entière est victime d'un préjudice (« Une voix intérieure me dit que je serais plus utile à ma patrie, si j'étais à la place que la raison et la philosophie m'assignent. [...] eh ! par quelle fatalité suis-je forcé de croupir dans l'oubli, tandis que je brûle du désir honorable d'utiliser mes moyens, et de prendre l'essor vers une route plus digne de moi $»^{59}$ ). S'il rappelle son statut nobiliaire ( « cœur bien né», issu d'une « classe d'hommes que l'on nommait privilégiés »), il ne cesse de mettre en valeur le principe méritocratique : les promotions successives dans sa carrière militaire sont liées à « l'estime de mes chefs, et la bienveillance de mes frères d'armes » à qui il apprend « à obéir sans élever le moindre murmure $»^{60}$. Au final, Rosny présente sa requête comme le produit d'une situation créée par un désordre qui heurte « la raison et la philosophie ». Au fil du texte, le premier Consul est présenté comme l'instance exclusive de contrôle des carrières : c'est lui qui, à travers les promotions individuelles, doit faire fonctionner la société et assurer l'ordre du jeu 
social. C'est là que le rôle joué par un acteur comme Rosny se révèle particulièrement important. Avec d'autres, il construit, légitime et diffuse les nouvelles formes de domination sociale et politique sur lesquelles Bonaparte s'appuie pour renforcer son pouvoir individuel et construire sa légende. Selon Rosny, le premier Consul doit rendre possible le " progrès » de chaque individu en permettant le développement de leurs qualités individuelles : «Tant que l'Anarchie a divisé la France, [il n'a] conservé aucun espoir d'avancement», mais avec le nouveau gouvernement, Rosny ose espérer la «justice» et les « moyens de développer quelques dispositions que la nature a placées en [lui] et qui peut-être un jour me concilieraient l'estime des chefs de l'État $»^{61}$. De productions en productions, Rosny n'a eu de cesse de critiquer les " libertés » et de solliciter les autorités pour qu'elles rétablissent l'ordre. En 1802, confronté à des difficultés et mécontent de son « exil » bourguignon, il participe à la vaste entreprise de propagande qui légitime le renforcement du pouvoir exécutif et l'abandon des principes républicains. Or, s'il ne parvient pas à convaincre le premier Consul de répondre favorablement à sa requête, Rosny cherche encore à s'adapter au nouveau contexte en investissant un nouveau genre de production et une nouvelle identité d'homme de Lettres, celle d'érudit.

\section{Le recours à l'érudition (1802-1814)}

Alors qu'il sollicite Bonaparte pour quitter la Bourgogne, il publie un ouvrage d'histoire locale consacré à Autun, Histoire de la ville d'Autun, connu autrefois sous le nom de Bibracte ${ }^{62}$. Cherchant sans doute à faire « de mauvaise fortune bon cœur », il valorise sa « retraite » en Bourgogne pour investir un nouveau domaine de production lié à l'histoire érudite. Ainsi, en 1804, il publie encore un Julius Sacrovir, ou le Dernier des $E^{\prime} d u e n s^{63}$. Cette étude, dédiée à Lucien Bonaparte et présentée comme la première pierre d'une vaste histoire nationale rattache son auteur à une tradition d'écriture de l'histoire propre à la culture nobiliaire et lui permet de s'inscrire dans l'intérêt grandissant porté à l'histoire des Celtes qui se cristallisera en 1805 à travers la création de l'Académie celtique ${ }^{64}$. Cet

(61) Ibid.,p. 8 .

(62) Joseph Rosny, Histoire de la ville d'Autun, connu autrefois sous le nom de Bibracte... par Joseph Rosny, Autun, impr. de Ph. Dejussieu, 1802, XXIV-352 p.

(63) Joseph Rosny, Julius Sacrovir, ou le Dernier des Éduens dédié à Lucien Bonaparte par Joseph Rosny, Paris, Frechet, 1804, XIII-282 p.

(64) Philippe Bourdin, « La part de l'histoire dans les éloges de la Nation. Académisme et Révolution ", Identités nationales dans l'Europe des Lumières, Siècles, Université Blaise Pascal, Clermont-Ferrand, 1999, p. 7-34. 
ouvrage est suivi l'année suivante de la publication d'un recueil de poésies du XVI ${ }^{\mathrm{e}}$ siècle, La Vie et la mort, poésies du XVI siècle, par Pierre. Matthieu ${ }^{65}$, annoté par Rosny et dédié cette fois à une autre grande personnalité de l'Empire, Regnaud de Saint Jean d'Angély. Alors que se multiplient les critiques contre les « petits » littérateurs accusés de participer au « déclin » de la littérature et que la valorisation du XVII ${ }^{\mathrm{e}}$ siècle devient un moyen de pourfendre les productions des Lumières et de la Révolution, Rosny cherche encore à bénéficier du soutien des autorités. Il développe à l'occasion de la publication de ce recueil de poésie une stratégie qui participe à la promotion du « Grand Siècle » et à la valorisation du modèle du mécénat royal du XVII ${ }^{e}$ siècle $^{66}$. Dans l' «Avertissement », il rappelle que « retiré à Autun », il avait le projet d'écrire l'histoire de cette ville et avait invité les « savants et les littérateurs de ce pays à contribuer au mérite de ce monument historique en [lui] donnant connaissance de tous les matériaux qu'ils pouvaient avoir entre les mains ». S'écartant progressivement de la figure de l'écrivain révolutionnaire, il rejoint celle de l'académicien d'Ancien Régime. C'est ainsi qu'il eut l'occasion de découvrir un ancien manuscrit anonyme qu'il entreprit de déchiffrer et de publier. Selon Rosny, ce manuscrit doit être attribué à Pierre Matthieu, « conseiller intime et historiographe du roi Henri IV » (« quoique plusieurs personnes l'attribuent à un certain François Perrin, ancien chanoine de l'église cathédrale d'Autun [...] mais plusieurs raisons [le] portent à rejeter cette opinion, comme étant dénuée de tout fondement »). Rosny ne fait rien au hasard; il a sans doute été informé qu'un exemplaire du même ouvrage a été opportunément découvert quelques mois auparavant dans la Bibliothèque impériale! Cette publication permet à Rosny de participer aux débats du moment qui divise le monde des Lettres, reconnaissant ainsi la supériorité de la littérature du $\mathrm{XVII}^{\mathrm{e}}$ siècle sur celle du XVIII et du début du XIX ${ }^{\mathrm{e}}$ siècle (" nos ouvrages modernes ne sont que d'aimables colifichets [...] absolument dénués de cette profondeur d'imagination, de cette force d'idées qui caractérise les ouvrages de nos aïeux »). Mais finalement l'essentiel n'est peut-être pas là... Il ajoute au début de l'ouvrage un «Précis historique de la vie de Pierre Matthieu » : issu d'un milieu roturier de province, son parcours ne saurait se comparer à celui de Rosny, et pourtant. Après ses études, Mat-

(65) Joseph Rosny, La Vie et la mort, poésies du XVI siècle, par P. Matthieu,... publiées et augmentées de notes et de commentaires, par Joseph Rosny, Paris, impr. des sciences et arts, an XIII-1805, 88 p.

(66) Jean-Luc CHAPPEY, « Le XVII ${ }^{\mathrm{e}}$ siècle comme enjeu philosophique et littéraire au début du XIX ${ }^{\mathrm{e}}$ siècle », Cahiers du Centre de recherches historiques, avril 2002, $\mathrm{n}^{\circ}$ 28-29, p. 101-116. 
thieu tente de se construire une réputation dans le monde des Lettres, recherche la protection d'un grand et se tourne - avec insuccès - vers le prince de Parme. Après cet échec, il achète une charge d'avocat du roi au présidial de Lyon et décide d'orienter sa production vers... l'histoire :

«Connaissant toutes les difficultés qu'un auteur doit surmonter pour percer la foule des écrivains obscurs, et sachant que les grandes réputations ne peuvent s'acquérir que par des travaux estimables et utiles à la société, Matthieu abandonna la poésie, dont il avait fait jusqu'alors ses plus chères délices, pour se lancer dans une carrière plus noble et plus élevée, celle de l'Histoire $»^{67}$.

Après plusieurs productions et bénéficiant de la protection d'une grande personnalité de l'administration royale qui l'introduit auprès d'Henri IV, Matthieu devient historiographe de France et acquiert à travers ses récits des différents règnes une réputation importante. C'est au lendemain de la mort d'Henri IV qu'il compose et publie les poésies sous le titre de «Tablettes du conseiller Matthieu». Il serait sans doute trop simpliste de postuler l'effet de miroir entre les parcours du conseiller d'Henri IV et celui de Rosny. Néanmoins, il faut se rappeler que le projet de recréer la fonction d'historiographe du roi (qui serait chargé de recueillir tous les matériaux « qui appartiennent à l'histoire de l'Empire français ») émerge dès 1805 et sera défendue par le ministre de l'Intérieur pour lutter contre la dégradation de la littérature avant d'être complètement enterré par l'empereur en avril $1807^{68}$. Si l'on peut penser que Rosny cherche encore à " publier » sa réputation et à gagner quelques bénéfices, on constate combien le rôle de ce type d'acteur a pu être important dans la légitimation du pouvoir impérial. Rosny ne cesse de publier des textes qui mettent en scène le système du mécénat et célèbrent la grandeur du Prince. De nouveau, Rosny en appelle à l'empereur pour intervenir dans l'espace des productions littéraires afin de rétablir l'ordre, l'État étant appelé à agir comme l'instance de consécration littéraire (le jugement du " public » étant - on le voit - déprécié). Par ce biais, Rosny intervient dans les débats essentiels concernant le statut de l'écrivain et les rapports entre les Lettres et les autorités, débats qui sont particulièrement importants au début de l'Empire comme l'illustre celui suscité lors du concours proposé par la seconde classe de l'Institut en 1805 sur « l'indépendance de l'homme de Lettres ». Or, en dépit de tous ses efforts, la reconnaissance

(67) Joseph Rosny, La Vie et la mort, op. cit., p. 11.

(68) Annie Jourdan, Napoléon, héros, imperator, mécène, op. cit, p. 236-238. 
comme « homme de Lettres » ne vient pas : dans son Rapport présenté à l'empereur en 1808, Marie-Joseph Chénier ne lui fait même pas l'honneur de le citer en dépit des productions érudites tels que son Tableau littéraire de la France pendant le XIII ${ }^{e}$ siècle ${ }^{69}$ et sa Notice historique sur Arnauld, abbé de Cîteaux ${ }^{70}$. Son activité ne s'arrête pourtant pas. En janvier 1810, alors qu'il est enfin revenu à Paris (il loge au $\mathrm{n}^{\circ} 10$, rue des Grès SaintJacques), il publie le Journal central des académies et sociétés savantes, un journal qu'il présente comme une réponse aux attentes liées à l'essor du nombre des sociétés savantes de provinces largement suscité par les autorités administratives ${ }^{71}$. Support de communications et d'échanges entre les diverses institutions savantes de la capitale et des provinces, ce journal se veut encore un instrument nécessaire à la « publication » des réputations. Au moment où l'espace intellectuel connaît des transformations profondes, Rosny cherche à ouvrir un espace de reconnaissance collective à des hommes de Lettres, savants de province en quête d'une notoriété parisienne :

« On a pu voir que son établissement n'a pour objet que d'encourager les efforts des savants et des littérateurs des départements, à qui souvent, pour jouir d'une grande réputation, il ne manque que la faculté de s'exercer sur un plus vaste théâtre. Aussi, est-ce pour obvier, autant que possible, à cet inconvénient, que nous nous attacherons, dans ce journal, à donner à leurs travaux toute la publicité dont ils sont dignes $\gg^{72}$.

Face à ceux qu'ils dénoncent comme des «littérateurs » et des « charlatans », il oppose la figure de «l'érudit». En 1811, il publie un Épitre à Voltaire dans les Champs-Élysées ${ }^{73}$ où rappelant ses apparte-

(69) Tableau littéraire de la France pendant le XIII siècle, ou Recherches historiques sur la situation des arts, sciences et belles lettres depuis l'an 1200 jusqu'en 1301, par Joseph de Rosny dédié à S. Ex. M. le comte Regnault de Saint Jean d'Angély,... Paris, J.-B.-D. Hécart fils, 1809, 280 p.

(70) Notice historique sur Arnauld, abbé de Cîteaux, légat du St Siège, archevêque de Narbonne, et célèbre écrivain du XIIr siècle, par Joseph de Rosny, Valenciennes, H.-J. Prignet, 1810, 19 p.

(71) Jean-Pierre Chaline, Sociabilité et érudition. Les sociétés savantes en France, Paris, CTHS, 1995, p. 36.

(72) Journal central des académies et des sociétés savantes, « Avertissement », p. 5.

(73) Épitre à Voltaire dans les Champs Élysées, H.J. Prignet, Valenciennes, 1811, 7 p. : «Associé correspondant de l'Académie celtique de Paris, de l'Académie Impériale des sciences et Belles-lettres de Gênes, de l'Académie Ionienne, de celles de Dijon, Caen, Besançon ; de l'Athénée de Niort ; de la Société Teylérienne à Harlem ; de la Société Philologique de Lille ; de celle d’Émulation de Cambrai; de la Société des sciences physiques et médicales d'Orléans; des sociétés d'agriculture de Douai, Chalons et Provins ; des Sociétés des sciences et arts de Nantes, Rouen, Lille, Autun, Macon, Le Mans, Alençon et secrétaire perpétuel de la Société libre des sciences, arts, commerces et industrie de Valenciennes »". 
nances aux diverses sociétés savantes et académies, il se tourne vers la figure tutélaire de Voltaire pour célébrer les «grands " écrivains et dénoncer, à l'instar d'un Rivarol en 1788, la « sottise » de ces « petits » littérateurs toujours plus nombreux :

« Depuis que tu n'es plus, les sots plus arrogants,/Enhardis par leur nombre et forts de ton absence,/Avec impunité lèvent la tête en France./ L'un, pesant rédacteur d'insipides écrits,/Pour illustrer son nom fait bailler tout Paris ; / L'autre, en beaux prospectus, délayant son génie,/Tient bureau de raison et de philosophie ; / Celui-ci, parfumé de quelques grains d'encens/Accordés par hasard à ses travaux naissants,/Déjà, de son grenier, croit régir le Parnasse ;/Celui-là, non moins fou, dans sa burlesque audace, /Rime tout ce qu'il dit, et ne parle qu'en vers/Ou prononce hardiment à tort et à travers ».

Il reste encore une trace de l'activité débordante de Rosny. Quelques semaines avant de mourir, il envoie encore un "Placet allégorique présenté à sa majesté Louis XVIII - par un ancien capitaine d'infanterie réformé en septembre dernier sans aucun traitement, après 27 ans de services », un texte qu'il adressera - en corrigeant le titre - à Napoléon quelques semaines plus tard. Sous la forme fantasmagorique, il construit encore un récit de justification du mécénat, célébrant le Prince en place ${ }^{74}$. Au terme de son existence, il réinvestit opportunément son identité nobi-

(74) Placet allégorique présenté à S. M. l'Empereur et Roi, par un officier réformé. [Signé : Joseph de Rosny.], Paris, impr. de Didot jeune, (s. d.), in-fol., VII p. (s.d.) : « On dit qu'alors il vint à vaquer dans les cieux / Certain emploi très fécond en richesses. / La discorde aussitôt se glisse entre les Dieux, / Les demi-dieux et surtout les déesses. / Brigues et factions se formant tour à tour, / Bientôt de Jupiter désunissent la Cour. [Face à cette situation, Jupiter se fâche :] « Que chacun devant moi ne parler qu'à son tour ; / Par un effet de ma clémence extrême, / Chacun aura sa voix, jusque au Dieu d'Amour : / Telle est ma volonté suprême. Que chaque protecteur flatte son protégé ;/ Qu'il soit, par ses bons soins, en savant érigé ; / Qu'il vante ses vertus, qu'il le transforme en sage, / J'y veux bien consentir, puisque tel est l'usage ; / Mais rappelez-vous tous qu'en briguant un emploi / Il faut citer des faits, et non pas un vain titre. / D'être juste, surtout, je m'impose la loi ». [Face aux concurrents divins, se dresse un « simple mortel»]: «D'un signe Jupiter l'invita d'approcher. / Son air était modeste, et son maintien timide, / Ce ne fut qu'en tremblant qu'il osa faire un pas : / - Quel est ton protecteur ? - Hélas, je n'en ai pas... / - Quel est ton appui ? - En vous seul il réside... / - Quoi briguer un emploi sans avoir de patron ! / Dit le père des Dieux, dans sa surprise extrême. / Parle-moi sans détour : tu crois donc en toi-même / Avoir bien consulté tes droits et la raison, / Et que te seuls talents sont d'abord ce qui frappe ? - Je ne m'en connais pas... Seigneur, si j'en avais, / Déjà l'on m'aurait vu comblé de vos bienfaits, / Car jamais à vos yeux le mérite n'échappe. / Sire, en moi vous voyez un faible prosateur / Qui fit pendant dix ans l'ingrat métier d'auteur... / - Jeune insensé, réponds : n'as-tu pas d'autre titre ? Longtemps dans les combats j'ai suivi le Dieu Mars, / Et d'une longue guerre affronté les hasards... / De mon sort à présent veuillez être l'arbitre. [Grâce à ce discours, le mortel obtient la place] Tu méritais l'emploi ; ta franchise l'obtient ; / C'est prouver qu'en ma cour l'intrigue est impuissante ». 
liaire et fait jouer son statut de militaire comme le montre l'extrait de cette petite lettre envoyée avec le manuscrit :

« J. de Rosny, ex capitaine, quartier maître, trésorier du $144^{e}$ régiment d'infanterie de ligne - note pour sa majesté en faveur du sieur Le Bailly-De Rosny. Le Bailly de Rosny, homme de Lettres, ex-capitaine d'infanterie et fils de M. de Rosny, ancien doyen des directeurs des fermes du Roi, après avoir parcouru pendant vingt ans tous les degrés du malheur, a été réformé sans aucun traitement le 27 septembre dernier par Napoléon, après 27 ans de service. Cet officier père d'une nombreuse famille, après avoir perdu comme tant d'autres, toute sa fortune à la révolution, se voit à la veille de manquer de l'absolu nécessaire. Il ne lui reste plus d'autre ressource que de se jeter aux pieds de son roi pour implorer de sa justice et de son humanité, soit une petite pension qui puisse le mettre à l'abri des horreurs du besoin, soit un emploi dans une administration quelconque. Son existence ne serait pas assez longue pour reconnaître un pareil bienfait $»^{75}$.

En conclusion, il n'est pas question de porter un jugement sur l'échec ou la réussite de Joseph Rosny et encore moins de gloser sur le caractère plus ou moins opportuniste de sa trajectoire. Comme beaucoup de ses contemporains, Rosny est un personnage «hybride» dont le parcours complexe et ambigu échappe aux catégories d'interprétation préconstruites. Là réside sans doute l'intérêt d'étudier ce type d'acteur dont l'identité ou la carrière ne se laissent pas facilement enfermer dans des modèles. Joseph Rosny dans la littérature, comme Bory de Saint Vincent ${ }^{76}$ dans les sciences, doivent justement lutter contre un processus de normalisation et de hiérarchisation qui caractérise l'espace intellectuel du début $\mathrm{du} \mathrm{XIX}^{\mathrm{e}}$ siècle, et trouver des cheminements originaux pour tenter de s'imposer comme écrivain ou savant. Loin d'être un simple acteur passif d'une évolution dont on connaît les grandes lignes (le fameux passage des «Lumières » au « Romantisme »), l'analyse du parcours de Rosny nous met aux prises avec les différentes respirations du monde des Lettres : de la littérature pédagogique aux Belles Lettres en passant par l'écriture de combat, les différentes étapes de la trajectoire de Rosny illustrent autant la complexité de l'histoire de la littérature pendant la période que les nécessaires adaptations d'un acteur qui cherche à s'imposer comme écri-

(75) Archives nationales, F/17/1025 - lettre de Rosny, 1814.

(76) Voir Hervé FerRière, Bory de Saint-Vincent (1778-1846), naturaliste, voyageur et militaire entre Révolution et Monarchie de Juillet. Essai biographique, thèse sous la direction de P. Corsi, Université de Paris I, novembre 2006. 
vain. La difficulté à définir ce statut d'écrivain (on voit là les limites de l'utilisation de la notion de « polygraphe ») est renforcée par celle face à laquelle on se trouve pour définir sa position sociale et politique : au regard de ses différentes "identités », il apparaît plus encore qu'on ne serait réduire son parcours à la seule «carrière » littéraire. Homme de Lettres, Rosny est aussi (et peut-être surtout) un militaire et un membre de l'administration. C'est à partir de cette constatation que l'on peut mesurer le rôle crucial joué par la pratique littéraire. Au fil de son parcours, l'écriture - considérée dans ses différentes formes - apparaît chez Rosny, non seulement comme une forme d'action relevant de logiques proprement littéraires, mais encore comme un support pour intervenir dans les espaces administratifs ou militaires. C'est à travers les usages des genres narratifs les plus divers que Rosny tente de rendre cohérente son identité et de construire l'unité de son «moi »" . Dans cette perspective, il est particulièrement représentatif d'une génération d'acteurs, contemporains d'une Révolution qui a profondément bouleversé l'ordre des identités : noble, militaire et écrivain puis administrateur et érudit, Rosny doit, comme beaucoup d'autres et toujours rapidement, s'adapter aux transformations sociales et politiques. Les critiques dont il a pu être la cible s'attaquent justement à ce caractère émietté, voire aberrant d'une identité qu' on ne peut apparemment pas réduire à une notice biographique et fondre ainsi dans les nouveaux modèles des types sociaux à partir desquels s'effectue la remise en ordre politique et sociale ${ }^{78}$. En attirant l'attention sur Joseph Rosny, il ne s'agit pas de rajouter le nom d'un écrivain inconnu au Panthéon littéraire, mais de mieux comprendre les processus qui rendent compte de ce statut d'écrivain "dédaigné et oublié », processus qui renvoient au final à la volonté de fermer le « livre de la Révolution » en occultant ceux qui en symbolisent les désordres.

Jean-Luc CHAPPEY

IHRF / Université de Paris I - Panthéon-Sorbonne

17, rue de la Sorbonne, 75005 Paris

jlchappey@gmail.com

(77) Gavin Edwards, Narrative Order, 1789-1819. Life and Story in an Age of Revolution, New York, Palgrave MacMillan, 2006 ; Damien ZANONE (dir.), Le Moi, l'Histoire 1789-1848, Ellug, Université Stendhal, Grenoble, 2005.

(78) Dominique Guillo, Les figures de l'organisation. Sciences de la vie et sciences sociales au XIX siècle, Paris, PUF, 2003. 